\title{
Analysis of a framework implementation of the transceiver performances for integrating optical technologies and wireless LAN based on OFDM-RoF
}

\author{
Adnan Hussein Ali ${ }^{1}$, Alaa Desher Farhood ${ }^{2}$, Maham Kamil Naji ${ }^{3}$ \\ ${ }^{1,3}$ Institute of Technology-Baghdad, Middle Technical University, Iraq \\ ${ }^{2}$ Technical Electronics Department, Technical Instructors Training Institute, Middle Technical University, Iraq
}

\begin{tabular}{l} 
Article Info \\
\hline Article history: \\
Received Aug 14, 2019 \\
Revised Feb 27, 2020 \\
Accepted Mar 8, 2020 \\
\hline
\end{tabular}

Keywords:

OFDM-RoF

Optical-wireless system

Optisystem

QAM

\begin{abstract}
The greatest advantages of optical fibers are the possibility of extending data rate transmission and propagation distances. Being a multicarrier technique, the orthogonal frequency division multiplexing (OFDM) can be applicable in hybrid optical-wireless systems design owing to its best spectral efficiency for the interferences of radio frequency (RF) and minor multipath distortion. An optical OFDM-RoF-based wireless local area network (W-LAN) system has been studied and evaluated in this work. The outline for integrating an optical technology and wireless in a single system was provided with the existence of OFDM-RoF technology and the microstrip patch antenna; these were applied in the Optisystem communication tool. The design of the proposed OFDM-RoF system is aimed at supporting mm-wave services and multi-standard operations. The proposed system can operate on different RF bands using different modulation schemes like 4.16 and 64QAM that may be associated to OFDM and multidata rates up to $5 \mathrm{Gbps}$. The results demonstrate the robustness of the integrated optical wireless link in propagating OFDM-RoF-based WLAN signals across optical fibers.
\end{abstract}

Copyright () 2020 Institute of Advanced Engineering and Science. All rights reserved.

\section{Corresponding Author:}

Adnan Hussein Ali,

Institute of Technology,

Middle Technical University,

Baghdad, Iraq.

Email: aaddnnaann63@gmail.com

\section{INTRODUCTION}

The past two decades have witnessed numerous advancements in mobile communication technology. This is evidenced in the access network extension services from voices to the data transmission, even to video transmission and other advanced bandwidth facilities. The next generation communication network is expected to provide high data rate with reduced latency. As the internet of things (IoT) and the era of big data are rapidly developing, wireless access network transmission bandwidth is seriously becoming an urgent issue that demands attention. With the conventional cellular structure, it had been tedious for achieving large capacity, high speed, and wide coverage areas; it will also be difficult to meet the requirements for future IoT, big data, and vehicle network. More attention has been given to the RoF technology due to the current effort in the research and expansion of certain key strategies; the RoF technology works can work in the mmwave band [1-3] as it is a kind of communication technology that 
combines photon with microwave; it also fully deploys high bandwidth and avoiding electromagnetic interferences attributes of the transmission of optical fiber. The emergence of microwave photonic technology could be beneficial in the transmission rate improvement of the of wireless access network via RoF technology.

Among the methods for photonic mmwave generation are optical heterodyne modulation, external modulation, and direct intensity modulation $[4,5]$. The direct laser is limited in bandwidth, hence, the production of up to $60 \mathrm{GHz}$ photonic mmwave via direct intensity modulation may be difficult. Regarding the optical heterodyne technique, it involves the transmission of the difference between the two frequencies and this is proportional to the essential mmwave frequency of the small linewidth optical waves that transmit the data for baseband transmission. The mmwave consists of a spectrum of extremely high frequencies (EHF) ranging from frequency bands of $30 \mathrm{GHz}$ to $300 \mathrm{GHz}$ [6]. A broad range of spectrum can be allotted to the mmwave band. Additionally, mmwaves are able to provide high data rates up to 10 Gbps and its spectrum allocation can be coordinated worldwide.

The RoF transmission system mainly consists of three sections namely, central station (CS), base station (BS) and optical distribution network (ODN) or optical fiber network (OFN) [7]. The implementation of mmwave bands in high capacity wireless systems via RoF technology has been proposed. According to Beans [8], the frequency around $60 \mathrm{GHz}$ attracts global telecommunication's interest because the frequency overlaps with the oxygen peak levels, i.e., the atmospheric attenuation of the frequency is $>15 \mathrm{DB} / \mathrm{Km}$ which allows cell size reduction (picocells) [9] and frequency reuse distance in cellular systems. Therefore, this factor increases the wireless capacity. Also, the frequency range opens the opportunity for the world-wide standardization and commercial production. The demand for mobile and internet services in areas that cannot be covered with the traditional wireless communication links keeps increasing. However, W-LAN signals distribution as per IEEE 802.11 standard is an attractive application for both outdoor and indoor systems [10].

\section{RADIO OVER FIBER SYSTEM (ROF)}

Among the major network explanations for high bandwidth in the wireless communication systems of the future is the optical fibers-based solutions for radio signals transmission between the BSs and MSC, $[11,12]$. This form of communication system is normally called a RoF solution as it requires the use of radio signal for the modulation of the intensity of an optical carrier. A simplified diagram of a RoF link is depicted in Figure 1, showing the conversion of the radio signal into an optical signal in an electronicto-optical (EO) converter at the CU. The transmission of the optical signal is via the fiber while its detection is at the BS where the original RF signal is recovered by an optical-to-electronic (OE) converter, amplified and transmitted from the BS antenna to the MS as shown in Figure 1. This optical carrier-based transmission of RF signals over fiber has several advantages compared to the traditional copper wire approach. The subsequent sections focused on some of the reasons why the RoF system is a proper approach for mobile communication signals transmission.

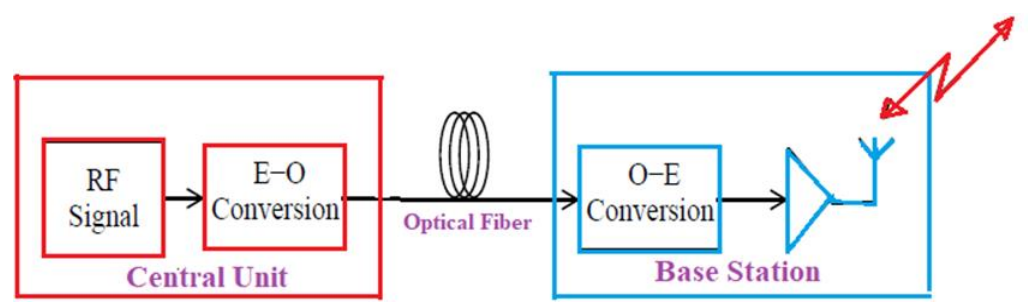

Figure 1. RoF link

Figure 2 describes an overview of subsystems of the RoF link. The extremely high radio frequency carrier wave is optically generated and is referred to as the photonic generation of millimeter wave. This optical carrier is utilized for optical data modulation of the transmitter data. The optically modulated data is transmitted through fiber-optic channel and the signal is detected at the receiver end. The detected signal is converted to electrical signal and the millimeter wave frequency signal is transmitted through the wireless channel $[13,14]$.

One of the major advantages of RoF communication is the high bandwidth of the optical fiber used. The third telecommunications window (TTW) ranges from $1530 \mathrm{~nm}$ to $1560 \mathrm{~nm}$; this is also referred to as 
the Erbium band and has a bandwidth of almost 3.8 THz. Studies are currently ongoing on how to exploit most of this bandwidth in both baseband and RoF communications [15]. Several DAs are spatially distributed in RoF-aided multicell network to encourage near-uniform coverage [16], but being that each DA can only cover a small area, the RF power required by each DA is low. This low RF power can easily be delivered without using amplifiers because of the low loss of optical fibers; hence, there will be increases in the RoF network power efficiency. Another advantage of using a low power at the DA is that it reduces inter-cell interference [17]. The RAUs employed in RoF communication system are simple in functionality but compact in size. Most RAUs are composed mainly of a PD, an amplifier, an electronic bandpass filter, and an antenna. When RF signals are to be propagated over a copper wire, there will be a need for an upconverter at the RAU; this requirement increases the complexity of the system, thereby increasing the cost [18]. Large scale usage of such RAU will increase the overall cost of the wireless network design.

In the network of the RoF transmission system that deals with simultaneous wireless multi mode procedure, the major components are one central station (CS), multiple mobile terminals (MTs) and multiple radio remote units (RRUs) [19]. Figure 3 depicts the block diagram of the CS. Below are the abbreviations for the functional modules in the system diagrams: continuous wave laser (CWL); polarization controller (PC); sine signal generator (SSG); Mach-Zehnder modulator (MZM); wavelength division multiplexing (WDM); erbium-doped optical fiber amplifier (EDFA); quadrature amplitude modulation (QAM); orthogonal frequency division multiplexing (OFDM); low pass cosine roll cutoff frequency (LPCROF)

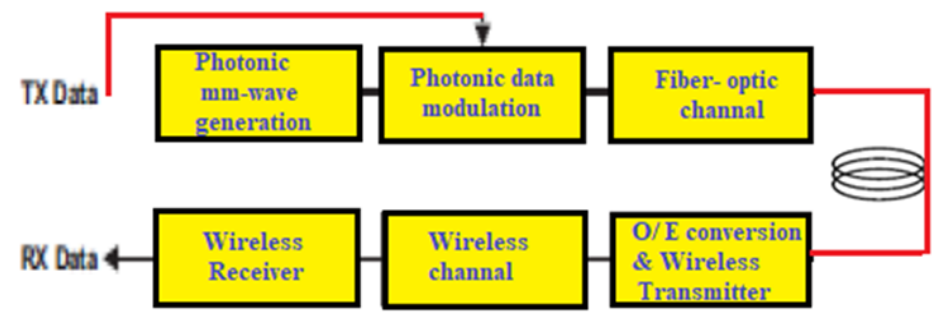

Figure 2. Block diagram of RoF subsystems

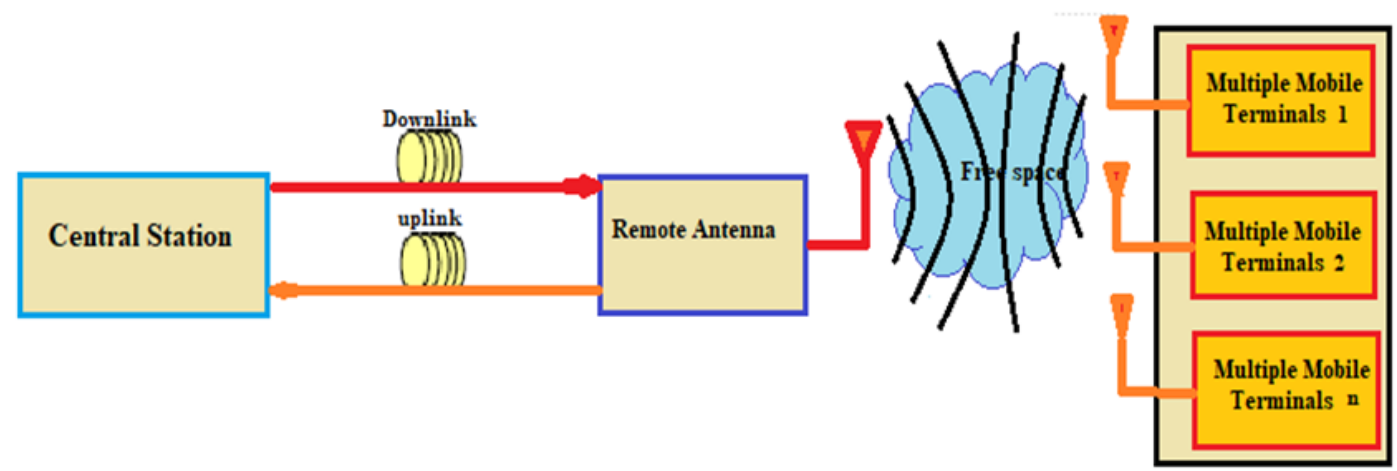

Figure 3. RoF transmission system network setup

\section{CONVENTIONAL OPTICAL-BASED WLAN SIGNAL}

This model is intended to propagate a modulated WLAN signal over an optical fiber prior to its wireless distribution via a microstrip patch antenna transmitter $T x$. The signal is received at the receiver while a QAM demodulator is used to demodulate the received signal. The main model variables are the optical fiber length and the free space distance.

In a simple intensity modulation direct detection system, low cost photodiodes and semiconductor lasers are preferred [20]. Due to the higher cost of external modulation it is not appropriate in such systems. It is relatively easy, cheap, and straightforward to implement frequency translation using low cost mixers and oscillators. The transfer of a low frequency reference tone from the CU to the RU can be implemented to lock the oscillators and ensure efficient frequency translation. Figure 4 illustrates the model of the system. The major components of a conventional optical wireless are the optical link components, a digital signal transmitter, a digital signal receiver, and the free link space-separated identical microstrip patch antennas. 


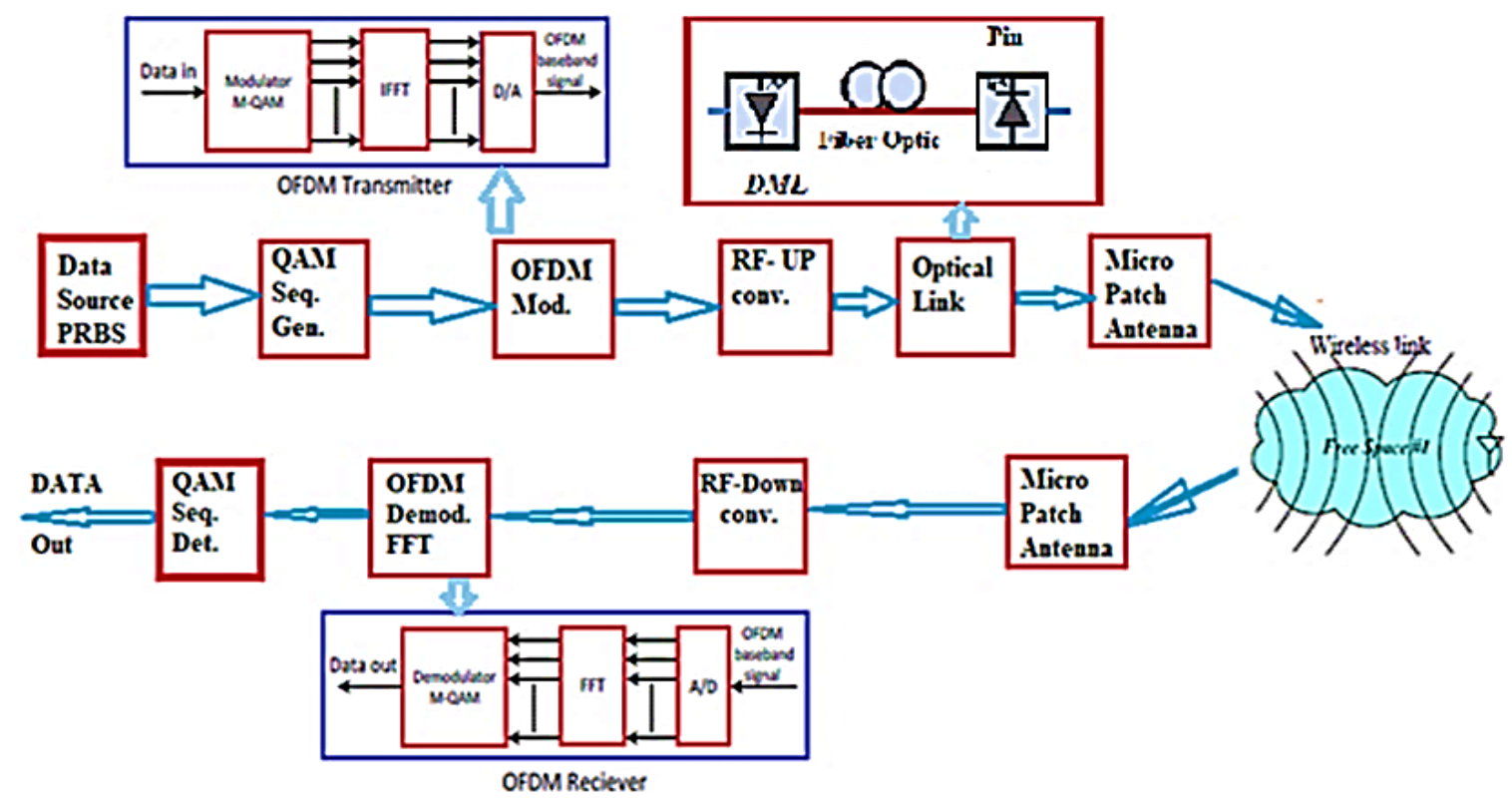

Figure 4. OFDM signal processing stages

\subsection{Transmitter system}

The generation of bits on the transmitter side is done using a pseudo-random bit sequence generator (PRBS) which generates a sequence of $2 \mathrm{k}-1$ length, where k represents the PRBS which can be computed thus:

\section{$K=\log ($ sequence length $) / \log (2)$}

Then, the bits sequence received from the PRBS with an order of $\mathrm{k}$ are mapped to the M-QAM constellation, with $M=2 n$ and $n$ being the number of bits/symbols. For instance, assume $n=2$, then, $M=4$, giving us 4-QAM, and 16-QAM for $n=4$, and 64-QAM for $n=6$. The bit sequence is split at the QAM output into 2 parallel subsequences. The multilevel pulses are generated by the M-ary pulse generator based on the M-ary signal input. Regarding a case of 4-QAM, two pulse levels (1 and -1) are generated, and each is transmittable in 2 quadrature carriers. At the quadrature modulator $(\mathrm{QM})$, the pulses are received before the implementation of a quadrature analog amplitude modulator by a QM. The modulation of the output signal is performed thus [20]:

$$
v_{\text {out }}(t)=G\left[I(t) \cos \left(2 \pm f_{c} t+c\right)-Q(t) \sin \left(2 f_{c} t+c\right)\right]+b
$$

where $I$ and $Q$ represent the input electrical signals; $G=$ the gain; $b=$ the bias which is set to 1 in this simulation while the radio frequency $f C$ is set to $2.4 \mathrm{GHz}$ (the same frequency as that of a microstrip antenna and it is the carrier phase).

The DML is employed in low-cost optics as a laser source for direct modulation; for direct detection, an InGaAs pin photodetector type is utilized, while an SMF is used as fiber optic link [21]. In optical communication system where there is a need for small size and low-cost components, it is not suggested to use optical amplifiers like semiconductor optical amplifiers (SOA) or EDFAs [22]. In an optical link, the photodetector mainly serves in receiving the fibers output optical power for onward detection and conversion into electrical current. The evaluation of the performance of a photodiode is mainly based on its responsivity $R$ and this metric is important as it portrays the generated photocurrent per unit optical power.

\subsection{Wireless system link}

As shown in Figure 4, the major components of a wireless link are the transmission and reception microstrip patch antennas with a free space link of varying distances " $d$ ". The transmission and reception antennas are similar as each one is a standard inset-fed microstrip that operates at $2.4 \mathrm{GHz}$ in an omni-directional radiation manner [23, 24]. The passive antenna can also be upgraded into an active antenna via integration with certain package components that consists of an RF low power amplifier and a band-pass filter. The bandwidth of the band-pass filter $(70 \mathrm{MHz})$ is sufficient to host the radio signal at that point. For the utilized RF amplifier, it is a broadband, general purpose amplifier. 


\subsection{System receiver}

As shown in Figure 4, the receiving antenna receives the RF signal at the receiving side prior to its filtering, amplification, and down-conversion using the QD. The data received from the QD is passed through an M-ray threshold detector which associates the electrical signal with decision instant determined by the user with a set of threshold levels [25]. During this comparison, the generation index of the output amplitudes is generated.

\subsection{OFDM}

Figure 4 shows the stages of the OFDM transmitter, its receiver in addition to optical link. The used modulation scheme during the simulations, as shown in the figure, is a QAM scheme. The 4-QAM symbols can be mapped into 52 OFDM-defined orthogonal subcarriers to form a vector that can serve as the input for the inverse fast Fourier transform (IFFT).

The transmitted OFDM signal can be mathematically expressed as follows:

$$
x_{m}=\frac{1}{\sqrt{N}} \sum_{k=0}^{N-1} X_{k}\left(e^{\frac{j 2 \pi k m}{N}}\right) \text {, for } 0 \leq m \leq N-1
$$

where $X k=$ the data symbols at $k$ th subcarrier; $\mathrm{N}=$ size of the IFFT and FFT. NSC refers to the number of OFDM subcarriers, and $N S C \leq N$. As shown in Figure 5, all the subcarriers are spaced equally by $\Delta f$.

\subsection{Antenna}

The two free space-separated identical microstrip patch antennas used with similar parameters and functionality are shown in Figure 6. The signal is filtered at the receiver side, then amplified and down-converted using the QDem before being filtered with a low-pass filter and subsequently reconverted to digital signal using an ADC.

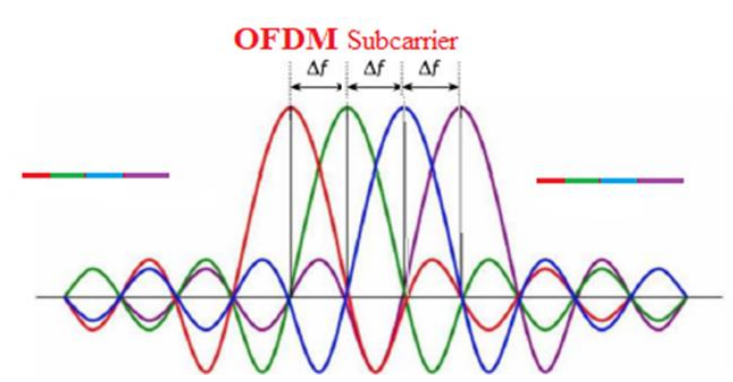

Figure 5. OFDM subcarrier with equal space interval $\Delta \mathrm{f}$ to reserve orthogonality

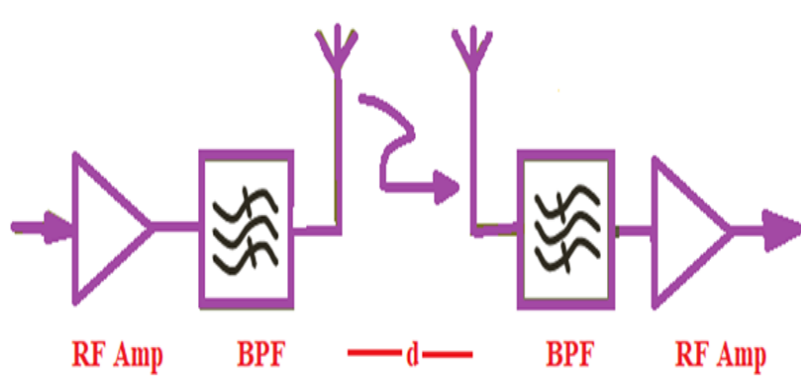

Figure 6. Two identical active microstrip patch antennas separated by a free space distance $(d)$

The discussions in this section focused on the optical OFDM down-link from the signal BRPS-generated signal via the reception of the signal at the end-user. However, this system differed from the previous modeling system by using the OFDM as a multicarrier modulation technique to process the signal rather than using only the QAM scheme obtainable in the traditional optical systems [26, 27]. Therefore, the following are the purpose for optical OFDM-RoF integration with wireless technology:

- The cost-effectiveness of the direct optoelectronic demodulation related to the RoF utilized for wireless communication.

- The spectral efficiency of OFDM signals which applied a single-sideband is high so that a limited chromatic dispersion transmission considered as low as well in RoF system.

- The fiber-RF remote arrangement in communication networks is important for spatial distribution microwave photonic where the optical modules that considered most actively are collected within the CS; also, neither EDFA nor independent laser source exists within the remote antenna units.

\section{SIMULATION AND RESULTS}

4.1. System model and parameters

The integrated optical OFDM wireless link is depicted in the block diagram shown in Figure 4. 6 which comprises of optical components, an OFDM transmitter and receiver, and 2 identical microstrips 
patch antennas that are separated by a modeled FSPL as shown in Figure 4. The data streams are first generated at the OFDM transmitter side using the PRBS in a similar manner as the traditional optical block diagram. The sketch of the steps involved in OFDM signal processing at different stages is shown in Figure 7. Optisystem communication tools are first used to build the blocks while Touchstone file format specifications are used to implement the wireless part of the designed the parameters of the patch antenna scattering in the two-port component. In the proposed model, the fiber lengths as well as the free space path are the major parameters that separate the microstrip patch antennas. The length of the SMF optical fiber ranges from $1 \mathrm{~km}$ to $100 \mathrm{~km}$ and is devoid of any component for optical amplification. The inter-antenna distance ranges from $40 \mathrm{~dB}$ to $80 \mathrm{~dB}$.

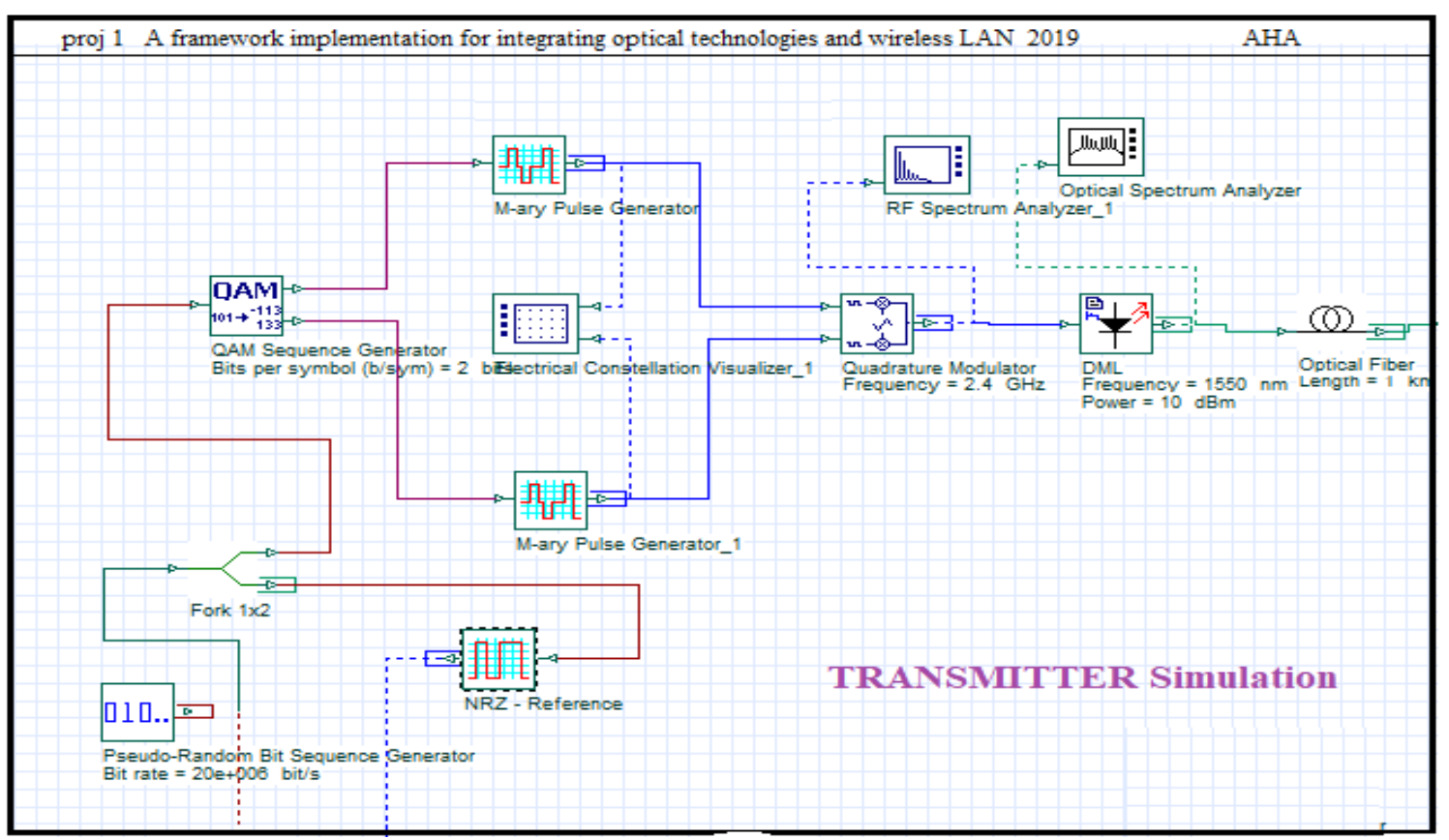

(a)

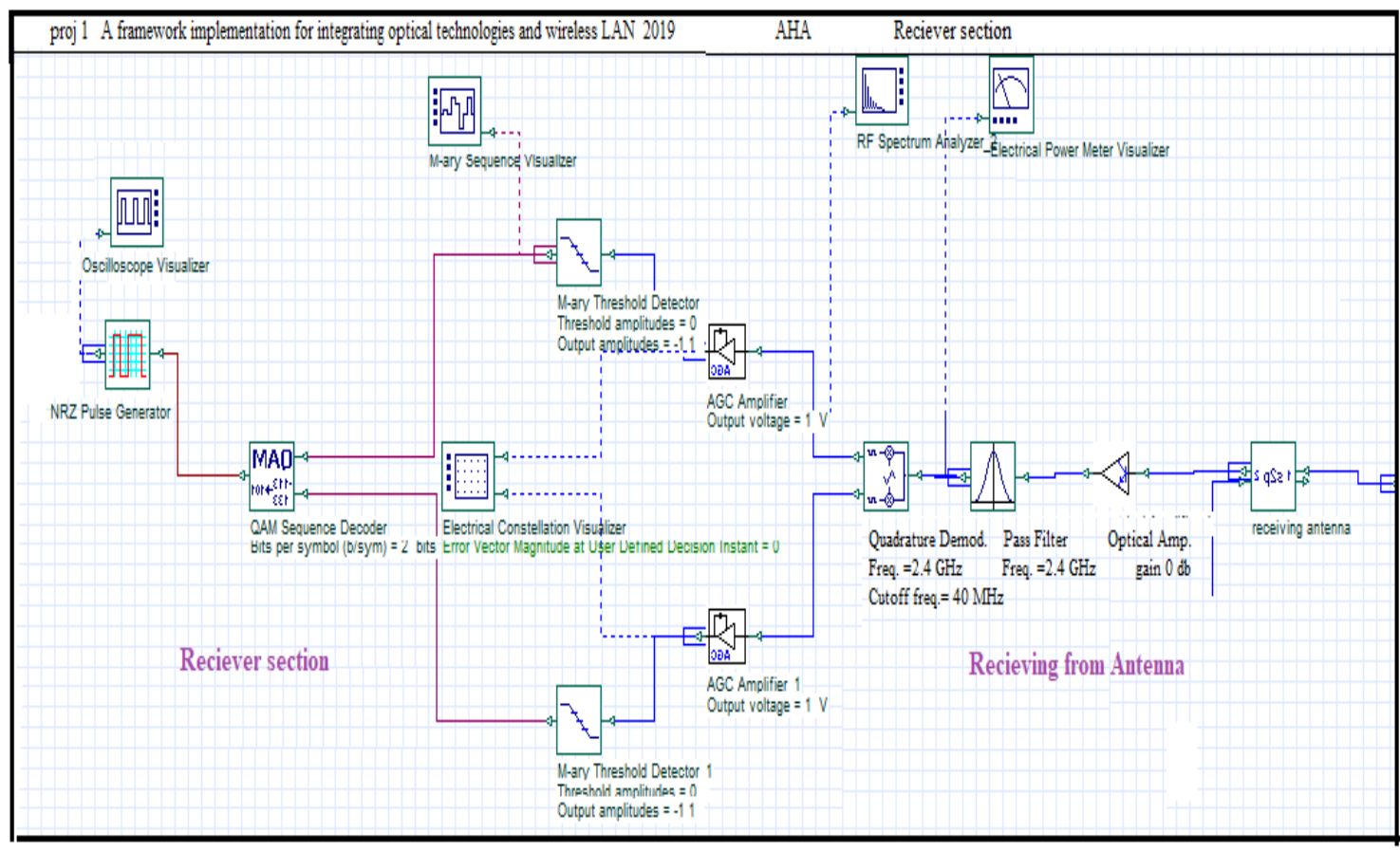

(b)

Figure 7. (a) transmitter, (b) receiver 
The sequence of $2^{11}-1$ length is generated by the PRBS; then, the received bits sequence from the PRBS is transformed into 4-QAM constellations (two bits/symbol) and these symbols are further converted into frames of $N$ parallel rows, representing the 48 subcarriers that carries data for a WLAN system. Furthermore, four pilot subcarriers were introduced into each frames string to get a total of 52 sub-carriers. This pilot is mainly aimed to be used for synchronization and channel equalization at the receiver if needed. Then, at the OFDM transmitter, the data stream in received in parallel rows.

\subsection{Simulation}

Optisystem communication tools were used to build al the blocks. Two main parameters were specifically used during the simulations in addition to the fiber length, launched optical power, and the free space in-between the microstrip patch antennas. The length of the SMF optical fiber ranged from 1-100 km without any component for optical amplification. The inter-antenna distance also varied from 40-80 dB.

Some of the achieved results were compared between the noise power values and receiving signals at many fibers lengths inter-antenna free space. The transmitted and received OFDM signals over the proposed integrated optically wireless link can be represented in Figure 8. The signal travelled 25, 50, and $100 \mathrm{~km}$ on the optical SMF before being received as seen in Figure 8 (b, c, and d); the signals still reserved their shapes even after $10 \mathrm{~m}$ range at free space propagation.

The signal spectrum at both ends after traveling $100 \mathrm{~km}$ along the SMF optical link at $50 \mathrm{~dB}$ free space propagation is shown in Figure 9. Figure 9(b) showed that the degradation in the received signal due to FSP attenuation. To validate the performance of the proposed design at $2.4 \mathrm{GHz}$, simulations were performed at different frequencies around the $2.4 \mathrm{GHz}$ central frequency to determine the region of maximum power reception. From Figure 9, it was evidenced that the maximum power was received at $2.4 \mathrm{GHz}$ radio center frequency, indicating maximum signal reception from the transmitting antenna.

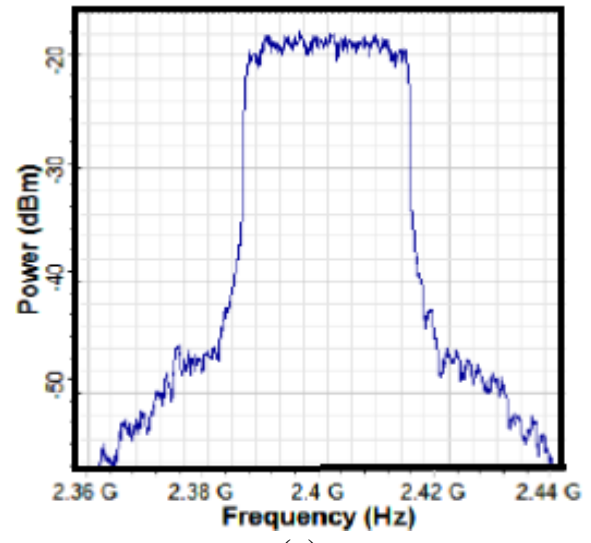

(a)

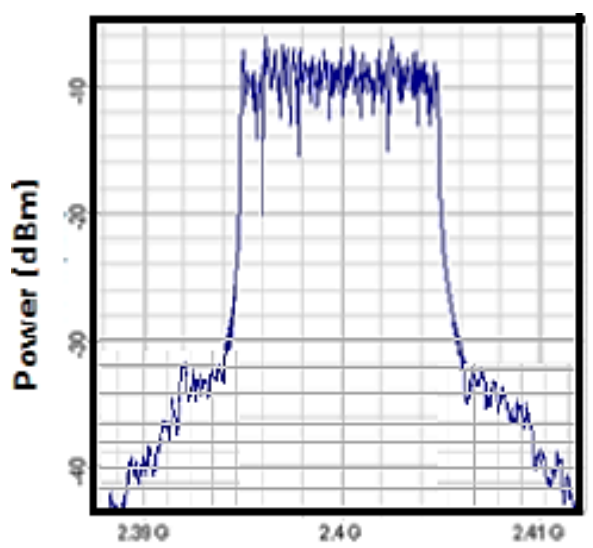

(c)

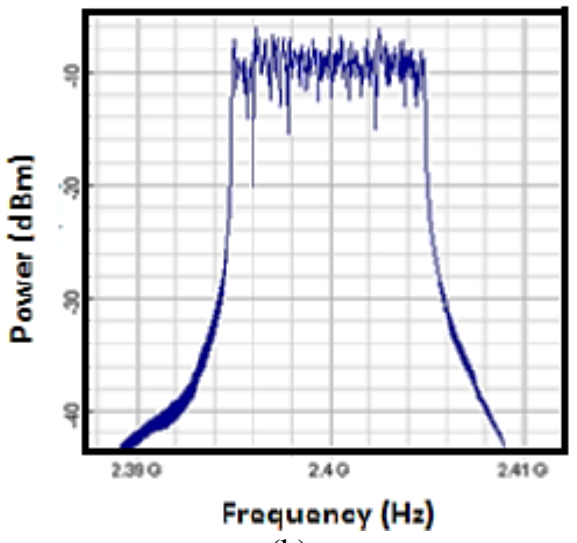

(b)

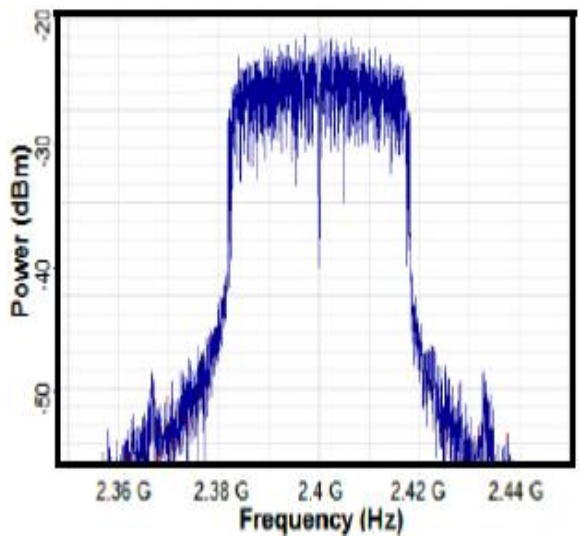

(d)

Figure 8. (a) OFDM Transmitting signal, (b) Receiving signal after $25 \mathrm{~km}$ fiber length $70 \mathrm{~dB}$ path loss, (c) Received signal after $50 \mathrm{~km}$ and (d) Spectrum at reception $100 \mathrm{~km}$ fiber length 

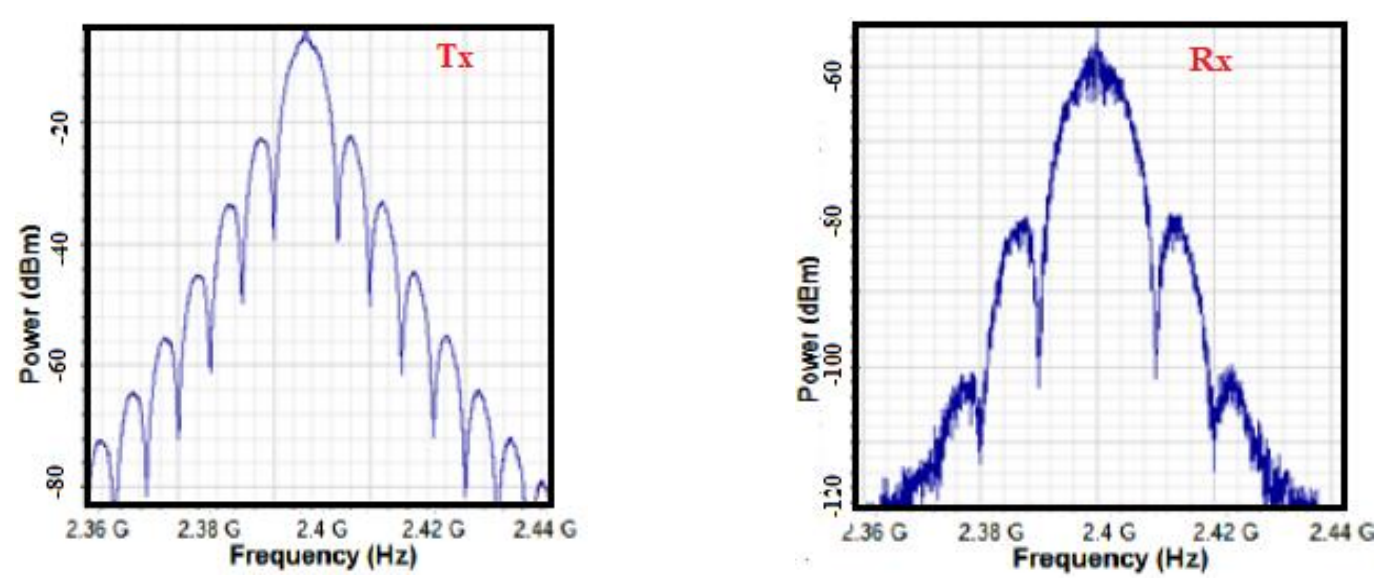

Figure 9. Transmitting and receiving antennas signal

\section{CONCLUSION}

From the outcome of this study, OFDM is considered suitable for wireless and optical communications combination for last mile purposes. The proposed optical OFDM-RoF system is designed and implemented utilizing Optisystem optical communication software tools. The designed cost-effective system can propagate OFDM signals utilized $2.4 \mathrm{GHz}$ RF over a gainful optical link since the signal is locally processed at the $\mathrm{CO}$ without the need for any amplifier. The simulation results showed the capability of the hybridized optical OFDM-active microstrip antenna system in improving the reliability of the system in terms of service coverage area. Thus, the designed multi-services RoF system is suitable for application in small cell concept and centralized management; it addresses the issues of flexibility and high throughput which are required in 5G small cell transport systems. Conclusively, the combination of optical OFDM based-WLAN and an active microstrip patch antenna can serve a distance of about $100 \mathrm{~km}$ on SMF and about 10 meters on the wireless platform.

\section{REFERENCES}

[1] El Yahyaoui, M., El Moussati, A., El Zein, G., \& Haddadi, K., "New millimeter wave generation scheme for MIMO-OFDM based Radio-over-Fiber system. Optics Communications, vol 442, pp. 101-105, 2019.

[2] Rebhi, S., Barrak, R., and Menif, M., "Flexible and Scalable Radio over Fiber Architecture," Radio engineering, vol. 27, no. 2, pp. 357-368. 2019.

[3] Al-Dabbagh, R. K., and Al-Raweshidy, H. S.,"64-GHz millimeter-wave photonic generation with a feasible radio over fiber system," Optical Engineering, vol. 56, no. 2, 2017.

[4] Ali, Adnan H., and Farhood, A., "Design and Performance Analysis of the WDM Schemes for Radio over Fiber System With Different Fiber Propagation Losses," Fibers, vol. 7, no. 3, 2019.

[5] Kareem, M. M., Ismail, M., Altahrawi, M. A., Arsad, N., Mansor, M. F., and Ali, Adnan H., "Grid Based Clustering Technique in Wireless Sensor Network using Hierarchical Routing Protocol," in IEEE 4th International Symposium on Telecommunication Technologies (ISTT), pp. 1-5, 2018.

[6] Adnan H. Ali, Hayder J. Alhamdane and Begared S. Hassen, "Design analysis and performance evaluation of the WDM integration with CO-OFDM system for radio over fiber system," Indonesian Journal Electrical Engineering and Computer Science (IJEECS), vol. 15, no. 2, 2019.

[7] Ahmed J. Abid, Fawzi M. Al-Naima, and Adnan H. Ali, "Comprehensive Modeling of PV Array based on Proteus Software," International Journal of Applied Engineering Research, vol. 13, no. 6, pp. 4440-4447, 2018.

[8] Badraoui, N., and Berceli, T., "Enhanced Capacity of Radio over Fiber Links Using Polarization Multiplexed Signal Transmission," in 20th Int. Conference on Transparent Optical Networks (ICTON), pp. 1-6, 2018.

[9] Ali, Adnan H., "Simultaneous Measurements for Tunable Laser Source Linewidth with Homodyne Detection," Computer and Information Science, vol. 4, no. 4, pp. 138-144, 2011.

[10] Tsai, C.-T., Lin, C.-H., Lin, C.-T., Chi, Y.-C., and Lin, G.-R., "60-GHz Millimeter-wave Over Fiber with Directly Modulated Dual-mode Laser Diode," Scientific Reports, vol. 6, no. 1, 2016.

[11] Ahmed J. Abid, Adnan Hussein Ali, "Smart Monitoring of the Consumption of Home Electrical Energy," International Journal of Computer Trends and Technology (IJCTT), vol. 47, no. 2, pp. 142-148, 2017.

[12] Yu, J., Li, X., and Chi, N., "Faster than fiber: over 100-Gb/s signal delivery in fiber wireless integration system," Optics Express, vol. 21, no. 19, pp. 22885-22904, 2013.

[13] A.H. Farhood, A.D., Naji, M.K., Rhaif, S.H., Ali, "Design and analysis of dual band integrated hexagonal shaped microstrip UWB antenna," Indonesian Journal Electrical Engineering and Computer Science (IJEECS), vol. 15, no. 1, pp. 294-299, 2019. 
[14] Kun Xu, Xiaoqiang Sun, Jie Yin, Hao Huang, Jian Wu, Xiaobin Hong, and Jintong Lin., "Enabling ROF Technologies and Integration Architectures for In-Building Optical-Wireless Access Networks," in IEEE Photonics Journal, vol. 2, no. 2, pp. 102-112, 2010

[15] Y. Niu, Y. Li, D. Jin, L. Su, and A. V. Vasilakos, "A survey of millimeter wave communications (mmWave) for 5G: opportunities and challenges,” Wireless Netw., vol. 21, no. 8, pp. 2657-2676, 2015.

[16] Borges, R. M., Reis Rufino Marins, T., Seda Borsato, M., Rodrigues Dias Filgueiras, H., and Cerqueira, A., "Integration of a GFDM-based 5G Transceiver in a GPON using Radio over Fiber Technology," Journal of Lightwave Technology, no. 99, pp. 1-1, 2018.

[17] C. Browning, A. Farhang, A. Saljoghei, N. Marchetti, V. Vujicic, L. E. Doyle, and L. P. Barry, "5G wireless and wired convergence in a passive optical network using UF-OFDM and GFDM," in IEEE Int. Conf. Communications Workshops (ICC Workshops), pp. 386-392, Paris, France, 2017.

[18] Adnan H. Ali, "Performance Evaluation of Wi-Fi Physical Layer Based QoS Systems on Fiber Using OPNET Modeler," International Journal of Soft Computing and Engineering (IJSCE), vol. 5, no. 3, 2015.

[19] Samir A. Lafta, Aktham H. Ali, Marwah M. Kareem, Yasser A. Hussein, Adnan H. Ali, "Performance simulation of broadband multimedia wireless networks simulation based on OPNET," Indonesian Journal Electrical Engineering and Computer Science (IJEECS), vol. 17, no. 1, pp. 1-9, 2020.

[20] M. A. Saad, Mustafa S. T., M. H. Ali, M. M. Hashim, M. Bin Ismail, and Adnan H. Ali, "Spectrum sensing and energy detection in cognitive networks," Indonesian Journal of Electrical Engineering and Computer Science (IJEECS), vol. 17, no. 1, pp. 465-472, 2020.

[21] Adnan H. Ali, Haeeder M. Noman, Hassan S. Hamad, "Analytical Execution of Dynamic Routing Protocols for Video Conferencing Applications Based on OPNET Modeler," International Journal of Engineering And Science, vol. 6, no. 3, pp. 49-56, 2017.

[22] H. Al-Raweshidy and S. Komaki, Eds., "Radio Over Fiber Technologies for Mobile Communications Networks, 1st ed., Artech House, Massachusetts, 2002.

[23] Mohammed, H.A., Ali, A.H., Mohammed, H.J., "The Affects of Different Queuing Algorithms within the Router on QoS VoIP Application using OPNET," International Journal of Computer Network and Communications, vol. 5, no. 1, pp. 117-124, 2013.

[24] P. T. Dat, A. Kanno, and T. Kawanishi, "Radio-on-radio-over-fiber: efficient fronthauling for small cells and moving cells," IEEE Wireless Commun., vol. 22, no. 5, pp. 67-75, 2015.

[25] Anas A. Hussien, Adnan H. Ali, "Comprehensive investigation of coherent optical OFDM-RoF employing 16QAM external modulation for long-haul optical communication system," International Journal Electrical and Computer Engineering (IJECE), vol. 10, no. 3, pp. 2607-2616, 2020,

[26] H. M. Noman, Ali A. Abdulrazzaq, Marwah M. Kareem, and Adnan H. Ali, "Improvement Investigation of the TCP Algorithms with Avoiding Network Congestion Based on OPNET," IOP Conference Series: Materials Science and Engineering, vol. 518, no. 5, 2019.

[27] M. K. Naji, A. D. Farhood, A. H. Ali, "Novel design and analysis of RF MEMS shunt capacitive switch for radar and satellite communications," Indonesian Journal of Electrical Engineering and Computer Science (IJEECS), vol. 15, no. 2, pp. 971-978, 2019. 